\title{
Lack of Association of the TP53BP1 Glu353Asp Polymorphism with Risk of Cancer: A Systematic Review and Meta-Analysis
}

\author{
Lei Liu ${ }^{1,5 *}$, Jinghua Jiao ${ }^{2}$, Yu Wang ${ }^{3}$, Dong Zhang ${ }^{4}$, Jingyang $\mathrm{Wu}^{1}$, Desheng Huang ${ }^{5}$ \\ 1 Department of Ophthalmology, The First Affiliated Hospital, China Medical University, Shenyang City, Liaoning Province, China, 2 Department of Anesthesiology, \\ Fengtian Hospital, Shenyang Medical College, Shenyang City, Liaoning Province, China, 3 Department of Development and Planning office, China Medical University, \\ Shenyang City, Liaoning Province, China, 4 Department of General Surgery, the Fourth People's Hospital of Shenyang, Shenyang City, Liaoning Province, China, \\ 5 Department of Epidemiology, School of Public Health, China Medical University, Shenyang City, Liaoning Province, China
}

\begin{abstract}
Objective: The TP53BP1 gene may be involved in the development of cancer through disrupting DNA repair. However, studies investigating the relationship between TP53BP1 Glu353Asp ( $r 560191$ ) polymorphism and cancer yielded contradictory and inconclusive outcomes. In order to realize these ambiguous findings, a meta-analysis was performed to assess the association between the TP53BP1 Glu353Asp (rs560191) polymorphism and susceptibility to cancer.

Methods: We conducted a search of all English reports on studies for the association between the TP53BP1 Asp353Glu (rs560191) polymorphism and susceptibility to cancer using Medline, the Cochrane Library, EMbase, Web of Science, Google (scholar), and all Chinese reports were identified manually and on-line using CBMDisc, Chongqing VIP database, and CNKI database. The strict selection criteria and exclusion criteria were determined, and odds ratios (ORs) with $95 \%$ confidence intervals $(\mathrm{Cls})$ were used to assess the strength of associations. The fixed or random effect model was selected based on the heterogeneity test among studies. Publication bias was estimated using funnel plots and Egger's regression test.

Results: A total of seven studies were included in the meta-analysis including 3,213 cases and 3,849 controls. The results indicated that the Glu353Asp ( $r 560191$ ) polymorphism in TP53BP1 gene had no association with cancer risk for all genetic models. In the subgroup analysis, the results suggested that Glu353Asp polymorphism was not associated with the risk of cancer according to ethnicity, cancer type, genotyping method, adjusted with control or not, HWE and quality score.
\end{abstract}

Conclusions: This meta-analysis suggested that the Glu353Asp (rs560191) polymorphism in TP53BP1 gene was not associated with risk of cancer.

Citation: Liu L, Jiao J, Wang Y, Zhang D, Wu J, et al. (2014) Lack of Association of the TP53BP1 Glu353Asp Polymorphism with Risk of Cancer: A Systematic Review and Meta-Analysis. PLoS ONE 9(3): e90931. doi:10.1371/journal.pone.0090931

Editor: William B. Coleman, University of North Carolina School of Medicine, United States of America

Received December 27, 2013; Accepted February 4, 2014; Published March 6, 2014

Copyright: $\odot 2014$ Liu et al. This is an open-access article distributed under the terms of the Creative Commons Attribution License, which permits unrestricted use, distribution, and reproduction in any medium, provided the original author and source are credited.

Funding: The authors have no support or funding to report.

Competing Interests: The authors have declared that no competing interests exist.

*E-mail: liuleijiao@163.com

\section{Introduction}

It was reported that there were about 12.7 million new cancer cases and 7.6 million cancer deaths through out the world in 2008 [1]. However, the etiology of cancer remains unknown and disease-modifying treatments are limited. In addition, since the involvement of cytokines in cancer was hypothesized, there were many candidate genes approaching in designing a case-control association study of single nucleotide polymorphisms (SNPs) including p53-binding protein 1 (TP53BP1).

TP53BP1 gene has played an important role in both DNA repair and cell cycle control and also mediates the DNA damage checkpoint through cooperation with damage sensors and signal transducers [2]. The TP53BP1 contains two BRCA1 C-terminal (BRCT) domains, which are essential for tumor suppressor functions [3]. The SNPs for TP53BP1 gene may play an important role in the etiology of cancer because of a direct role of TP53BP1 in the cellular response to DNA damage. Previous researches have revealed that no association between TP53BP1 Asp353Glu (rs560191) SNPs and cancer risk [4-9], but Kiyohara et al. reported that the Glu/Glu genotype of TP53BP1 Asp353Glu was associated with a decreased risk of lung cancer [10]. So the results of studies concerning association between Asp353Glu (rs560191) polymorphism in TP53BP1 gene and risk of cancer are conflicting.

Considering a single study may lack the power to provide a reliable conclusion, we performed a meta-analysis on these eligible studies to investigate the precise relationship between TP53BP1 Asp353Glu (rs560191) polymorphism and susceptibility to cancer, which would have a much greater possibility of reaching reasonably strong conclusions.

\section{Methods}

Selection of Eligible Studies

We searched Medline (US National Library of Medicine, Bethesda, MD), Embase, the Cochrane Library, Chinese Biolog- 
Table 1. Scale for quality assessment.

\begin{tabular}{ll}
\hline & \\
\hline Paramete & Score \\
\hline Source of cases & 2 \\
\hline Selected from population o rcancer registry & 1 \\
\hline Selected from oncology department or cancer institute & 0 \\
\hline No description & \\
Representativeness of controls & 2 \\
\hline Population-based & 1.5 \\
\hline Population-hospital mixed & 1 \\
\hline Hospital-based & 0 \\
\hline No description & \\
\hline Diagnosis of cancer & 2 \\
\hline Histological or pathologically confirmed & 1 \\
\hline Patient medical record & 0 \\
\hline No description & \\
\hline Specimens of cases for genotyping & 2 \\
\hline Peripheral blood or normal tissues & 1 \\
\hline Tumor tissues or exfoliated cells & 0 \\
\hline No description & 2 \\
\hline Quality control of genotyping & 2 \\
\hline Different genotyping assays confirmed the result & 1 \\
\hline Quality control by repeated assay & 0 \\
\hline No description & 2 \\
\hline Total sample size & \\
\hline$>1000$ & \\
\hline $200-1000$ & \\
\hline$<200$ & \\
\hline & \\
\hline
\end{tabular}

doi:10.1371/journal.pone.0090931.t001

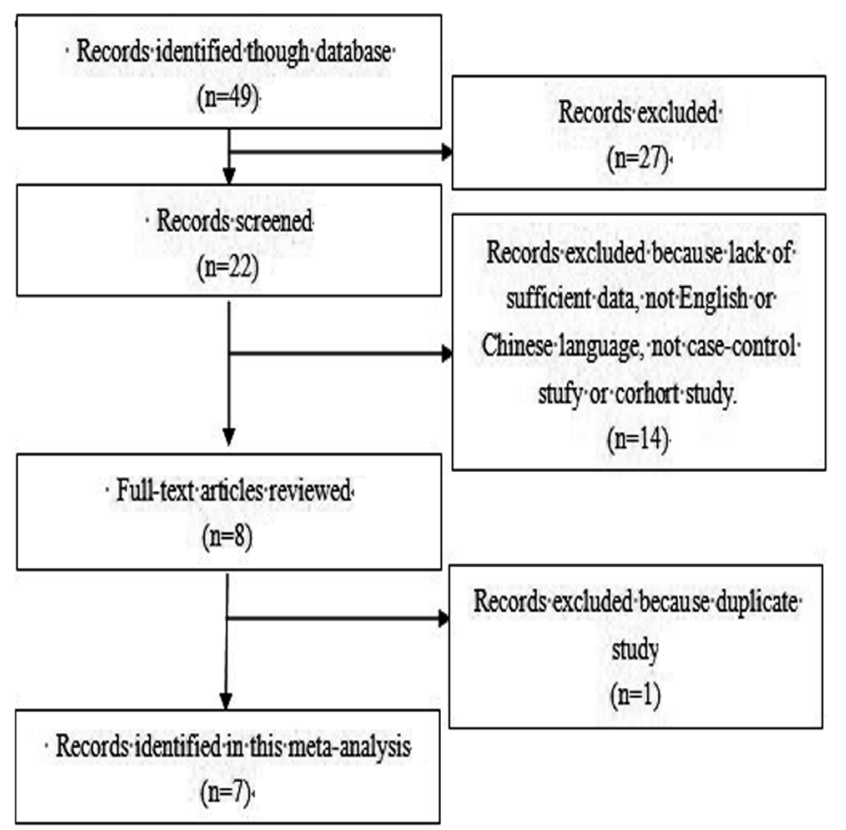

Figure 1. Flow chart demonstrating those studies that were processed for inclusion in the meta-analysis.

doi:10.1371/journal.pone.0090931.g001

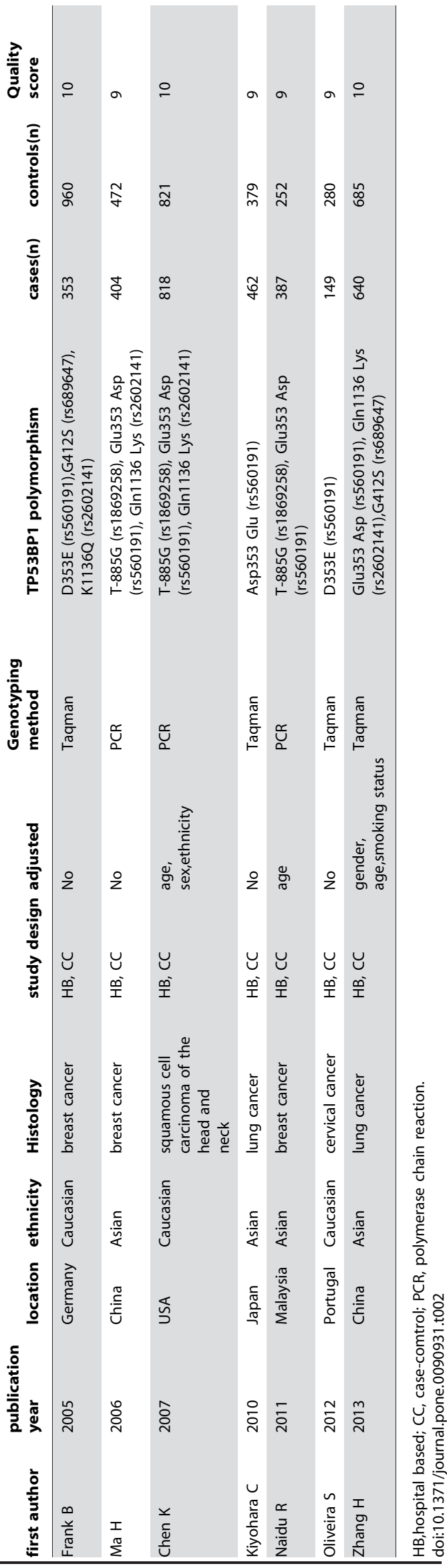




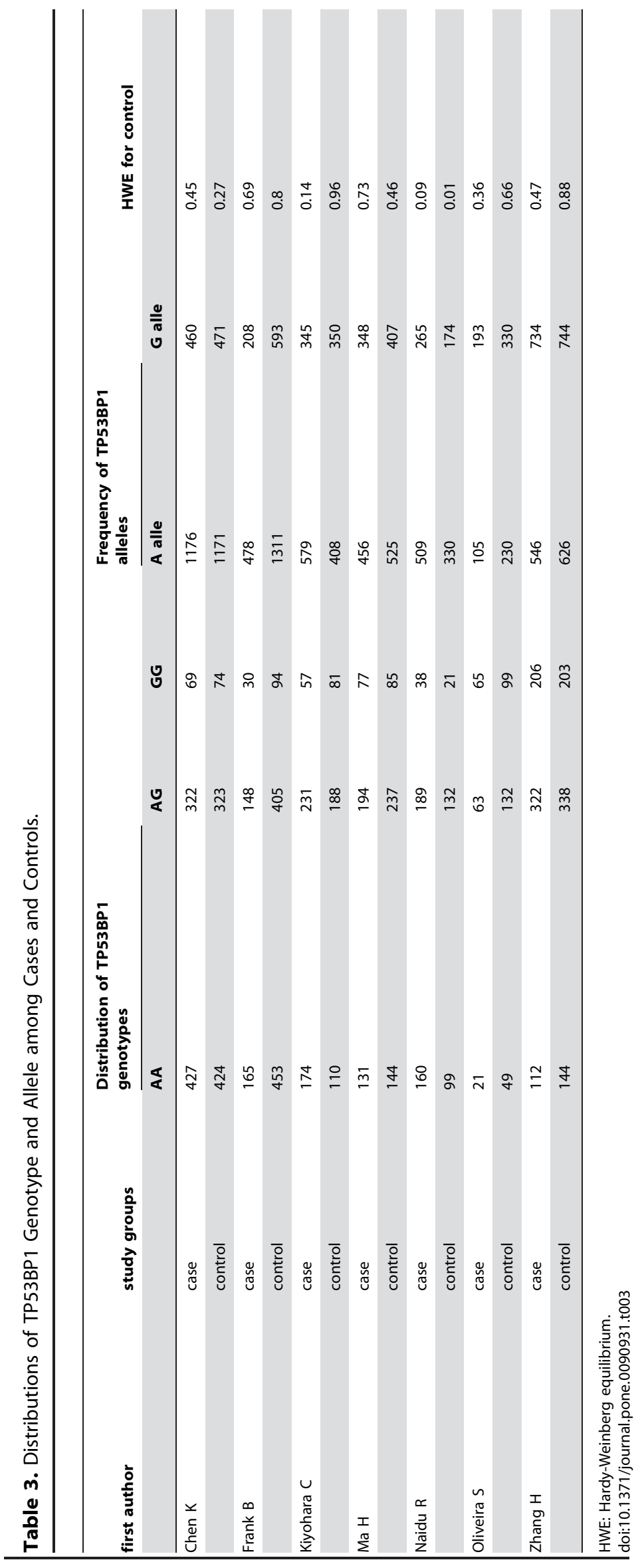




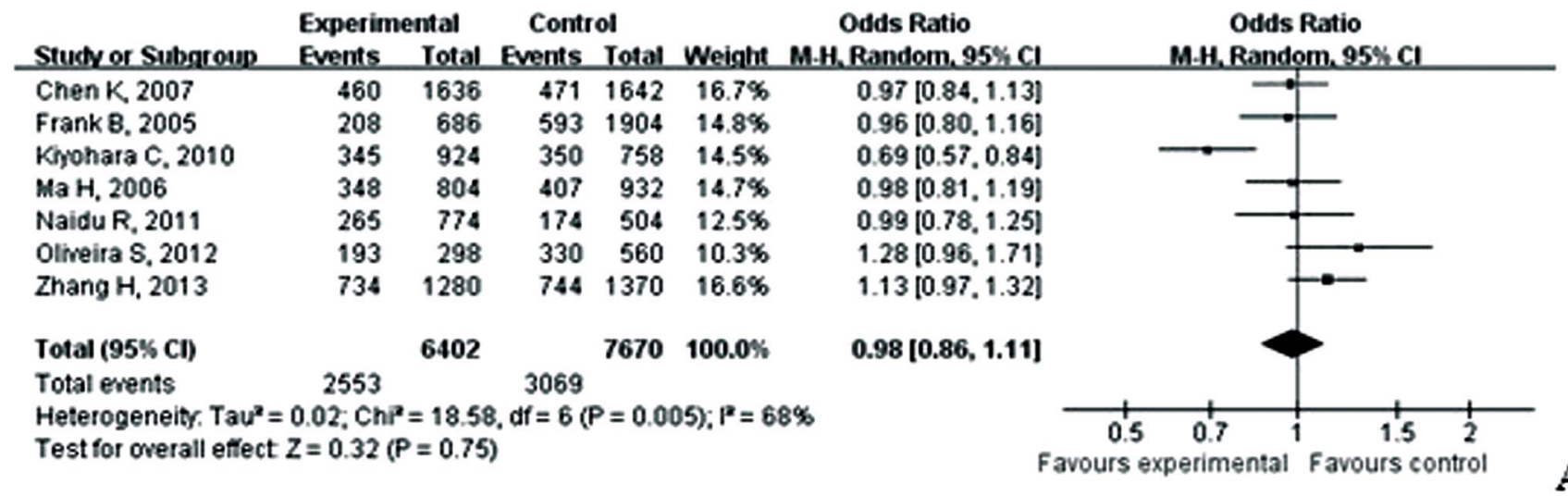

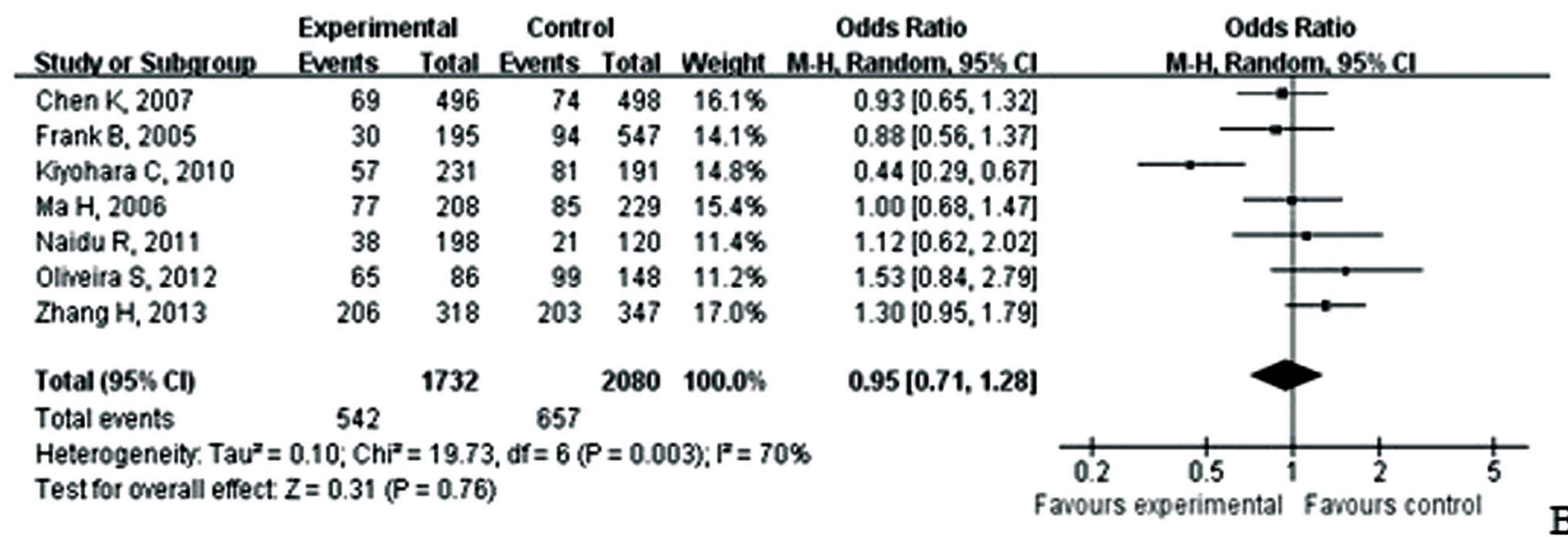

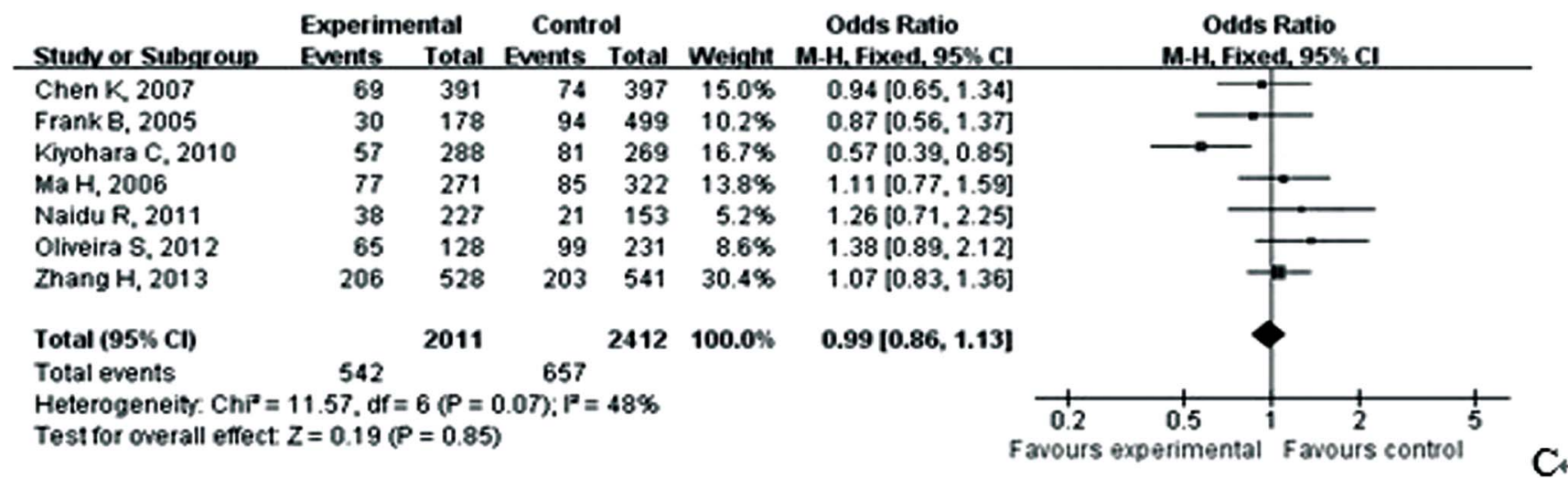

Figure 2. A. Forest plot of the association between cancer and the Glu353Asp (rs560191) mutation in overall population (G vs A). Figure 2.B. Forest plot of the association between cancer and the Glu353Asp (rs560191) mutation in overall population (GG vs AA). Figure 2.C. Forest plot of the association between cancer and the Glu353Asp (rs560191) mutation in overall population (GG vs AG).

doi:10.1371/journal.pone.0090931.g002

ical Medicine, China National Knowledge Infrastructure, Wang Fang Data and Chongqing VIP database (Last search was updated on December 20, 2013) using the terms "p53-binding protein 1 or TP53BP1 or 53BP1", "Asp353Glu or rs560191 or D353E", "cancer or tunor or carcinoma" and "polymorphism, variant or mutation". The selection was done without restriction on language, but we only included published articles written in English or Chinese. We used the PubMed option "Related Articles" for each study to retrieve additional potentially relevant articles. Reference lists were checked and researchers were contacted for additional literatures.

\section{Selection Criteria}

Studies were selected if they met the following criteria: (1) association study with a case-control or cohort design; (2) the study investigated the association between TP53BP1 (rs560191) polymorphism and the risk of cancer; (3) in the case of multiple publications from the same study group, the most complete and recent results were used.

\section{Exclusion Criteria}

The exclusion criteria were defined as: 1) abstracts, reviews and animal studies; 2) useless data reported, genotype number or 


\section{Experimental Control Odds Ratio Odds Ratio}

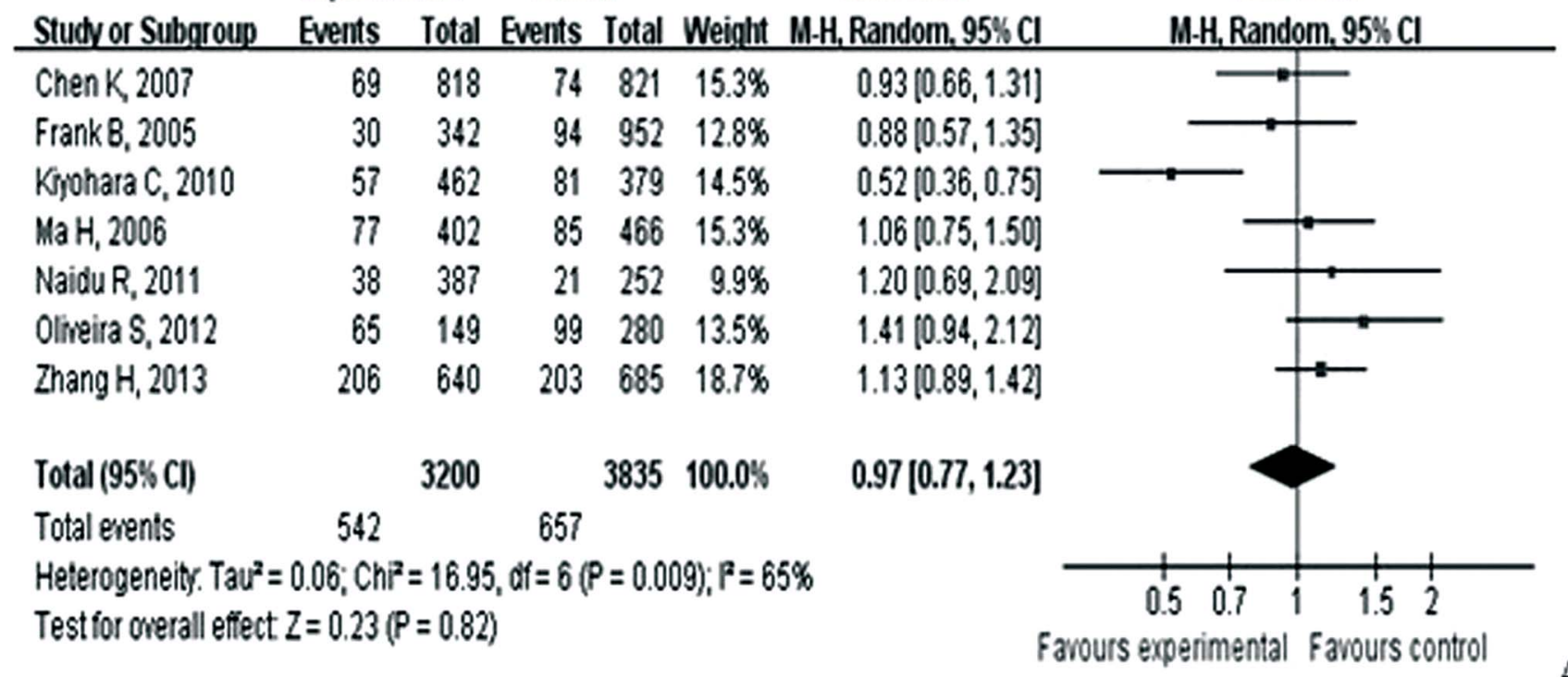

\section{Experimental Control Odds Ratio Odds Ratio}

\begin{tabular}{|c|c|c|c|c|c|c|c|}
\hline Studv or Subgroup & Events & Total & Events & Total & Weight & M.H. Fixed. $95 \% \mathrm{Cl}$ & M.H. Fixed, $95 \% \mathrm{Cl}$ \\
\hline Chen $\mathrm{K}_{2} 200 ?$ & 391 & 818 & 397 & 821 & $28.2 \%$ & $0.98[0.81,1.19]$ & \\
\hline FrankB, 2005 & 178 & 342 & 499 & 952 & $17.3 \%$ & $0.99[0.77,1.26]$ & \\
\hline Kysohara C, 2010 & 288 & 462 & 269 & 379 & $15.2 \%$ & $0.68[0.51,0.91]$ & \\
\hline $\mathrm{MaH}_{2} 2006$ & 271 & 402 & 322 & 466 & $13.3 \%$ & $0.93[0.69,1.23]$ & \\
\hline Naidu R, 2011 & 227 & 387 & 153 & 252 & $10.5 \%$ & $0.92[0.66,1.27]$ & \\
\hline Olneira S, 2012 & 128 & 149 & 231 & 280 & $3.1 \%$ & $1.29[0.74,2.25]$ & \\
\hline Zhang $H_{1} 2013$ & 528 & 640 & 541 & 685 & $12.5 \%$ & $1.25[0.95,1.65]$ & \\
\hline Total $(95 \% \mathrm{Cl})$ & & 3200 & & 3835 & $100.0 \%$ & $0.96[0.87,1.07]$ & \\
\hline Total events & 2011 & & 2412 & & & & \\
\hline $\begin{array}{l}\text { Heterogeneity. } \mathrm{Ch}^{2}= \\
\text { Test for orerall effect }\end{array}$ & $\begin{array}{l}10.51, d f= \\
z=0.69 / P\end{array}$ & $6(P=0$ & $10) ; l^{2}=$ & $43 \%$ & & & $\begin{array}{lll}5 & 0.7 & 1\end{array}$ \\
\hline
\end{tabular}

Test for orerall effect $Z=0.69(P=0.49)$

Figure 3. A. Forest plot of the association between cancer and the Glu353Asp (rs560191) mutation in overall population (GG vs AG+AA). Figure 3.B. Forest plot of the association between cancer and the Glu353Asp (rs560191) mutation in overall population (GG+AG vs AA). doi:10.1371/journal.pone.0090931.g003

frequency not included; and 3) study without sufficient data for meta-analysis. If more than one study was published by the same authors using the same case series, only the most recent study or the study with the largest size of samples was included in our metaanalysis.

\section{Data Extraction}

Two reviewers (Lei Liu and Jinghua Jiao) independently scrutinized studies on the associations between TP53BP1 Asp353Glu (rs560191) polymorphism and risk of cancer. When discrepancies were appeared, all investigators were recruited to assess the data. The following information was collected: First author, publication year, location, ethnicity, sample sizes of patients and controls, study design and genotype numbers.

The reviewers developed a quality assessment scale (Table 1), which was modified from previous studies [11-13], to evaluate the quality of eligible studies.

The review and analysis were guided to conduct by the PRISMA statement for preferred reporting of systematic review and meta-analysis [14].

Statistical Analysis

Odds ratios (ORs) with 95\% confidence intervals (CIs) for genotypes and alleles were used to assess the strength of association 


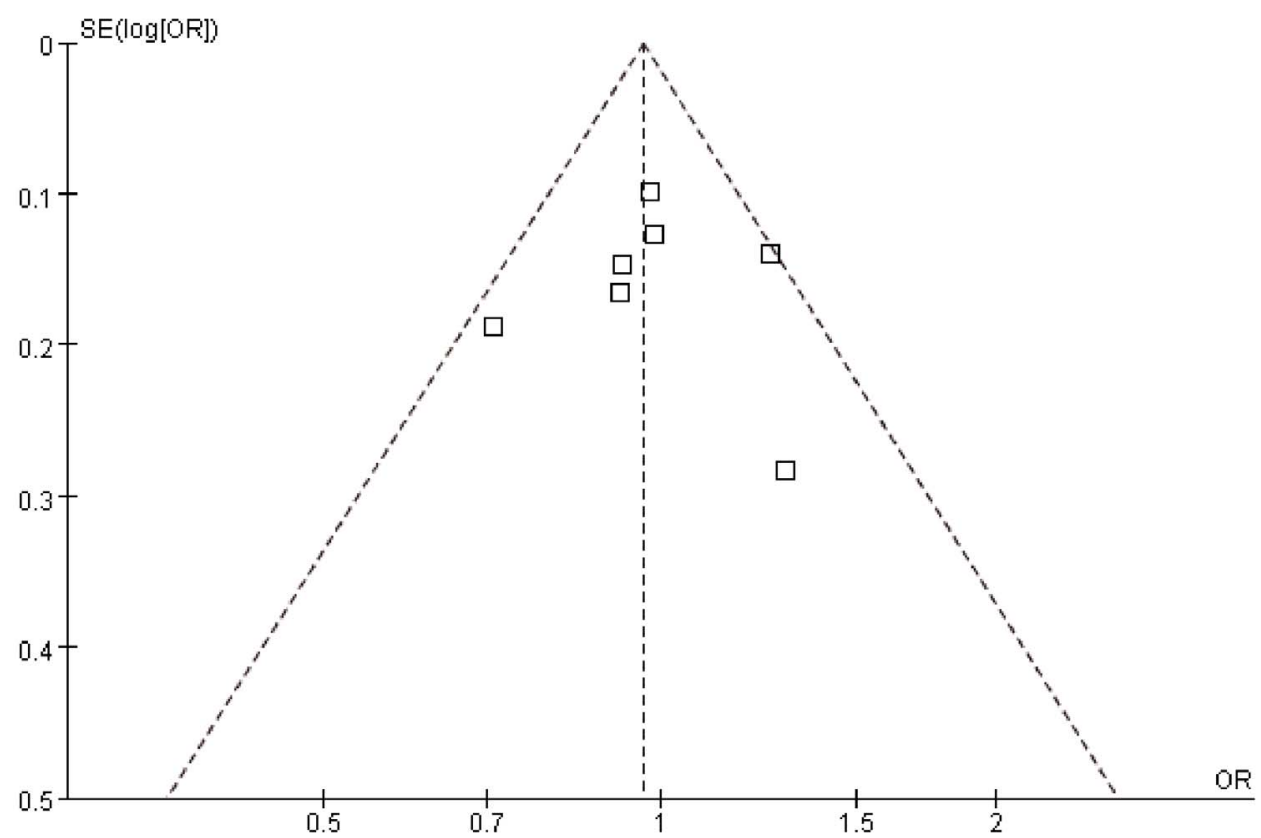

Figure 4. Funnel plot analysis on the detection of publication bias in the meta-analysis of the associations between Glu353Asp (rs560191) mutation and cancer risk.

doi:10.1371/journal.pone.0090931.g004

between TP53BP1 Asp353Glu (rs560191) polymorphism and risk of cancer. The ORs were performed for the allele contrasts, additive genetic model, as well as recessive genetic model and dominant genetic model, respectively. Heterogeneity was examined with $I^{2}$ statistic interpreted as the proportion of total variation contributed by between-study variation. We also measured the effect of heterogeneity using a quantitative measure, $I^{2}=100 \% \times(\mathrm{Q}-\mathrm{d} \mathrm{f}) / \mathrm{Q}$. If there was a statistical difference in terms of heterogeneity $\left(\mathrm{P}<0.10, I^{2}>50 \%\right)$, the random effects model would be used to estimate the pooled ORs $[15,16]$. Otherwise, the pooled ORs were estimated by the fixed effects model [17]. Sensitivity analysis was carried out by deleting one single study each time to examine the influence of individual data set on the pooled ORs. The possible publication bias was assessed with funnel plots and Egger's test. An asymmetric plot suggests a possible publication bias and the $\mathrm{P}$ value of Egger's test less than 0.05 was considered representative of statistically significant publication bias [18]. All statistical tests were performed with RevMan version 5.0 (Review Manager, Copenhagen: The Nordic Cochrane Centre, The Cochrane Collaboration, 2010) and Comprehensive Meta-Analysis software version 2.0 (Biostat, Englewood Cliffs, I.N.J., USA). $P$ value of smaller than 0.05 for any test was considered to be statistically significant.

\section{Results}

\section{Study Inclusion and Characteristics}

As showed in Figure 1, a total of seven studies were included in this meta-analysis including 3,213 cases and 3,849 controls [4-10]. The studies identified and their main characteristics were summarized in Table 1 and Table 2. Genotype distribution of six studies polymorphism did not differ from Hardy-Weinberg equilibrium with in control groups (all were greater than 0.05, Table 3).

\section{Quantitative Data Synthesis}

As showed in Table 4, meta-analysis of the total studies showed that there was no association between Asp353Glu (rs560191) polymorphism and risk of cancer under all five genetic models in overall population $(\mathrm{OR}=0.98,95 \% \mathrm{CI}=0.86-1.11$ for $\mathrm{G}$ versus $\mathrm{A} ; \mathrm{OR}=0.95,95 \% \mathrm{CI}=0.71-1.28$ for $\mathrm{GG}$ versus $\mathrm{AA} ; \mathrm{OR}=0.99$, $95 \%$ CI $=0.86-1.13$ for $\mathrm{GG}$ versus $\mathrm{AG} ; \mathrm{OR}=0.97,95 \%$ $\mathrm{CI}=0.77-1.23$ for recessive model; $\mathrm{OR}=0.96 ; 95 \% \mathrm{CI}=0.87-$ 1.07 for dominant model) (Figure 2 and Figure 3). In the subgroup analysis according to ethnicity, cancer type, adjusted with control or not, genotyping methods, HWE and quality score, the results suggested that Asp353Glu (rs560191) polymorphism were not associated with the risk of cancer. There was no significant publication bias according to Begg's and Egger's tests (Begg, $\mathrm{p}=0.21$; Egger, $\mathrm{p}=0.64$ ) and funnel plot (Figure 4).

\section{Sensitivity Analysis}

According to sensitivity analysis, the results showed us that there was no substantial modification of our estimates after exclusion of individual studies, indicating that the results were stable (data not shown).

\section{Discussion}

It is well known that SNPs may contribute to an individual's susceptibility to cancer and TP53BP1 is a key component in the cellular response to DNA damage [19]. Therfore, the SNPs of TP53BP1 may play an important role in the etiology of cancer. The conclusion that TP53BP1 gene played an important role in DNA repair has been well-researched, but the functional relevance of TP53BPl gene polymorphism has not been reported. It is possible that the sequence variation in the promoter and coding region of TP53BP1 might affect its transcription and downstream biological function $[4,5]$.

To the best of our knowledge, some researches that aim at the role of Asp353Glu (rs560191) polymorphism in cancer risk have 


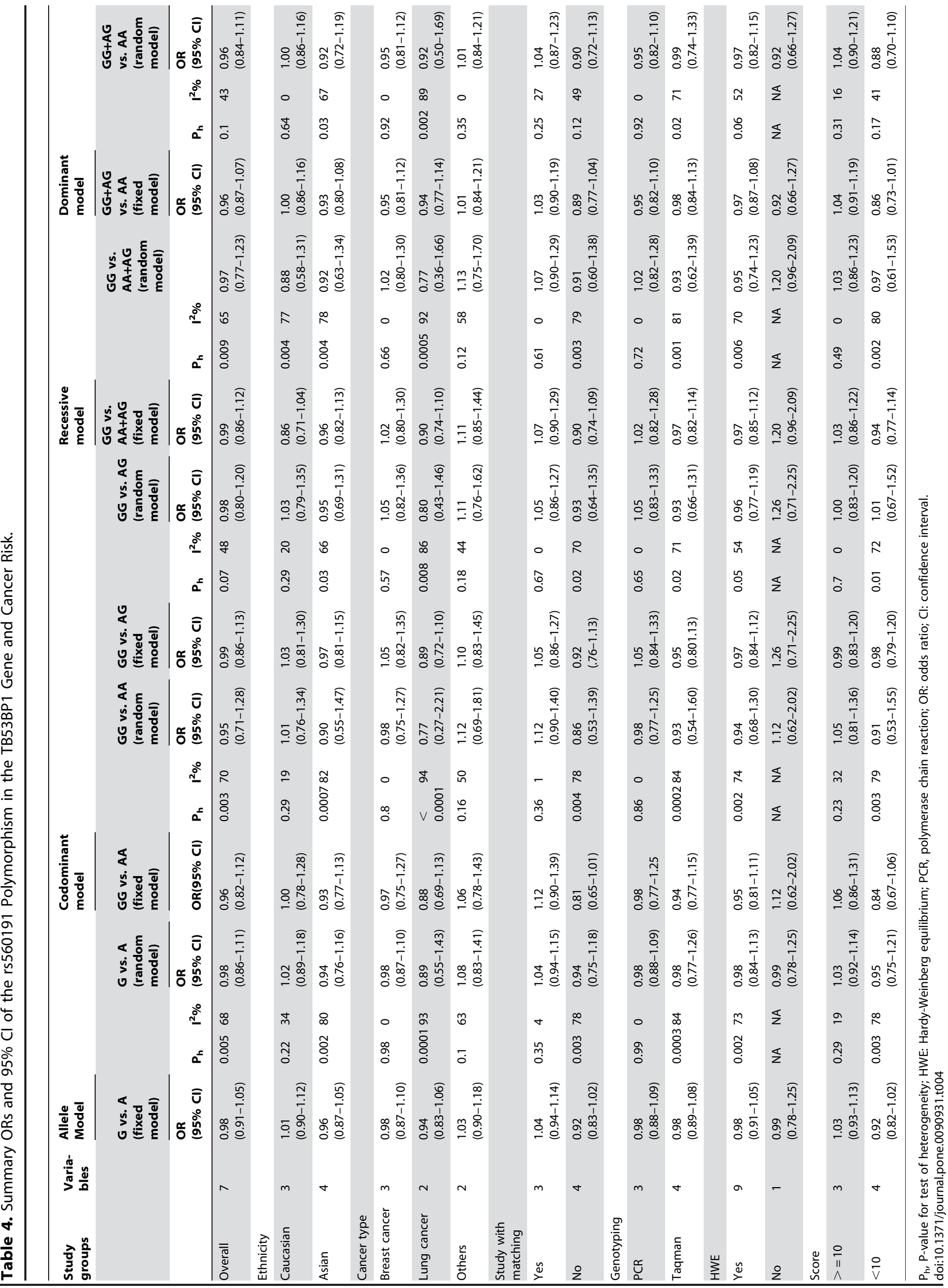


been performed, but the results are controversial. In order to evaluate on the association between the Asp353Glu (rs560191) polymorphism and cancer risk, we performed this meta-analysis.

We have not found a sinificant association between TP53BP1 Asp353Glu (rs560191) polymorphism and cancer risk in overall population, but different ethnicity, study design, genotyping methods and cancer type would be responsible for the negtive conclusions. We perfomed subgroup analysis based on these factors. However, the resluts showed us that Asp353Glu (rs560191) polymorphism were not associated with the risk of cancer according to ethnicity, cancer type, study with matching or not, genotyping methods, HWE and study score. That may be because only one study [10] reported that the Asp353Glu polymorphism was associated with a risk of cancer. Therefore, further studies are needed to confirm our results.

Some studies indicate that TP53BP1 variants may have protective effects on squamous cell carcinoma of the head and neck (SCCHN) risk but such effects were confined to TP53 Arg72Pro variant allele/haplotype carriers [5,8]. As the reason for few studies were perfomed and there were many meta-analysis related on TP53 Arg72Pro polymorphism and cancer risk [20,21], we could not use meta-analysis to analyze the relationship between TP53BP1 Asp353Glu (rs560191) polymorphism combined with TP53 gene polymorphism and cancer. In addition, Rudd et al. [22] and Truong et al. [23] found that Asp353Glu (rs560191) polymorphism was associated with lung cancer risk, but this association was not been found in the study [24] by Brooks JD et al. In addition, because lack of sufficient data from these three studies, we could not include these studies in this meta-analysis. That may be another reason for the negtive conclusion in this meta-analysis.

The meta-analysis by Timofeeva et al. [25] did not show a significant association between rs560191 polymorphism and lung cancer risk. It came to the same conclusion with our study. However, it was only concerned lung cancer risk. In our metaanalysis, the association between rs560191 polymorphism and other cancer types including cervical cancer, breast cancer and squamous cell carcinoma of the head and neck was also analyzed.

\section{References}

1. Ferlay J, Shin HR, Bray F, Forman D, Mathers C, et al. (2010) Estimates of world wide burden of cancer in 2008: GLOBOCAN 2008. Int J Cancer 127: 2893-2917.

2. Miwa S, Tome Y, Yano S, Hiroshima Y, Uehara F, et al. (2013) Single cell timelapse imaging of focus formation by the DNA damage-response protein 53BP1 after UVC irradiation of human pancreatic cancer cells. Anticancer Res 33: 1373-1377.

3. Williams RS, Green R, Glover JN (2001) Crystal structure of the BRCT repeat region from the breast cancer-associated protein BRCA1. Nat Struct Biol 8: 838-842.

4. Naidu R, Har YC, Taib NA (2011) Genetic polymorphisms of TP53-binding protein 1 (TP53BP1) gene and association with breast cancer risk. APMIS 119: 460-467.

5. Chen K, Hu Z, Wang LE, Zhang W, El-Naggar AK, et al. (2007) Polymorphic TP53BP1 and TP53 gene interactions associated with risk of squamous cell carcinoma of the head and neck. Clin Cancer Res 13: 4300-4305.

6. Ma H, Hu Z, Zhai X, Wang S, Wang X, et al. (2006) Joint effects of single nucleotide polymorphisms in P53BP1 and p53 on breast cancer risk in a Chinese population. Carcinogenesis 27: 766-771.

7. Frank B, Hemminki K, Bermejo JL, Klaes R, Bugert P, et al. (2005) TP53binding protein variants and breast cancer risk: a case-control study. Breast Cancer Res 7: R502-505.

8. Zhang H, Hao S, Zhao J, Zhou B, Ren Y, et al. (2013) Common Genetic Variants in 53BP1 Associated with Non-small Cell Lung Cancer Risk in Han Chinese. Arch Med Res doi: 10.1016/j.arcmed.2013.10.011. [Epub ahead of print].

9. Oliveira S, Ribeiro J, Sousa H, Pinto D, Baldaque I, et al. (2012) Genetic polymorphisms and cervical cancer development: ATM G5557A and p53bpl C1236G. Oncol Rep 27: 1188-1192.
There are several limitations in this meta-analysis that should be considered. First, cancer is a multi-factorial disease including complex interactions from environmental exposure to gene factors. In this meta-analysis, we had insufficient data to perform an evaluation of such interactions for the independent role of TP53BP1 Asp353Glu (rs560191) polymorphism in cancer development. Second, only seven studies were included in this metaanalysis. Thus, more studies are needed to identify this association more comprehensively. Third, study by Naidu et al. [4] showing genotype distributions of the control population that were not in HWE was included in this meta-analysis. Forth, we did not consider studies published in languages other than English/ Chinese or data presented in abstracted form; thus, publication and potential language biases may occur.

In conclusion, this meta-analysis suggested that the polymorphism in TP53BP1 Asp353Glu (rs560191) gene could not be regarded as a genetic risk factor for cancer. At the same time, this result should be interpreted cautiously. To verify this result, large scale case-control studies with detailed individual information are needed.

\section{Supporting Information \\ Checklist S1 PRISMA 2009 Checklist. (DOC)}

\section{Acknowledgments}

Thanks to Jingpu Shi, Ph.D, Professor of the Department of Clinical Epidemiology and Evidence Medicine in the First Affiliated Hospital of China Medical University. Thanks to the editors and anonymous reviewers.

\section{Author Contributions}

Conceived and designed the experiments: LL JHJ DZ. Performed the experiments: LL JHJ JYW YW DZ. Analyzed the data: DSH LL JHJ. Contributed reagents/materials/analysis tools: DZ LL. Wrote the paper: LL JL YW.

10. Kiyohara C, Horiuchi T, Miyake Y, Takayama K, Nakanishi Y (2010) Cigarette smoking, TP53 Arg72Pro, TP53BP1 Asp353Glu and the risk of lung cancer in a Japanese population. Oncol Rep 23: 1361-1368.

11. Liu GY, Jiang DK, Shen SQ Yu L (2011) MDM2 SNP309T. $>$ G polymorphism with hepatocellular carcinoma risk: a meta-analysis. Arch Med Res 42: 149-155.

12. Camargo MC, Mera R, Correa P, Peek RM Jr, Fontham ET, et al. (2006) Interleukin-1 beta and interleukin-1 receptor antagonistgene polymorphisms and gastric cancer: a meta-analysis. Cancer Epidemiol Biomarkers Prev 15: 1674 1687

13. Gao LB, Pan XM, Li LJ, Liang WB, Zhu Y, et al. (2011) RAD 51135G/C polymorphism and breast cancer risk: a meta-analysis from 21 studies. Breast Cancer Res Treat 125: 827-835.

14. Moher D, Liberati A, Tetzlaff J, Altman DG, The PRISMA Group (2009) Preferred reporting items for systematic reviews and meta-analyses: the PRISMA statement. PLoS Med 6: e1000097.

15. DerSimonian R, Laird N (1986) Meta-analysis in clinical trials. Control Clin Trials 7: 177-188.

16. DerSimonian R, Kacker R (2007) Random-effects model for meta-analysis of clinical trials: an update. Contemp Clin Trials 28: 105-114.

17. Mantel N, Haenszel W (1959) Statistical aspects of the analysis of data from retrospective studies of disease. J Natl Cancer Inst 22: 719-748.

18. Egger M, Davey SG, Schneider M, Minder C (1997) Bias in meta-analysis detected by a simple, graphical test. BMJ 315: 629-634.

19. Haffty BG, Goyal S, Kulkarni D, Green C, Vazquez A, et al. (2011) Evaluation of single nucleotide polymorphisms (SNPs) in the p53 binding protein 1 (TP53BP1) gene in breast cancer patients treated with breast-conserving surgery and whole-breast irradiation (BCS+RT). Int J Radiat Oncol Biol Phys 80: 385391. 
20. Weng Y, Lu L, Yuan G, Guo J, Zhang Z, et al. (2012) p53 codon 72 polymorphism and hematological cancer risk: an update meta-analysis. PLoS One 7: e45820.

21. Zhao L, Zhao X, Wu X, Tang W (2013) Association of p53 Arg72Pro polymorphism with esophageal cancer: a meta-analysis based on 14 case-control studies. Genet Test Mol Biomarkers 17: 721-726.

22. Rudd MF, Webb EL, Matakidou A, Sellick GS, Williams RD, et al. (2006) Variants in the GH-IGF axis confer susceptibility to lung cancer. Genome Res 16: 693-701.
23. Truong T, Sauter W, Mc Kay JD, Hosgood HD 3rd, Gallagher C, et al. (2010) International Lung Cancer Consortium: coordinated association study of 10 potential lung cancer susceptibility variants. Carcinogenesis 31: 625-633.

24. Brooks JD, Teraoka SN, Reiner AS, Satagopan JM, Bernstein L, et al. (2012) Variants in activators and downstream targets of ATM, radiation exposure, and contralateral breast cancer risk in the WECARE study. Hum Mutat 33: 158164.

25. Timofeeva MN, Hung RJ, Rafnar T, Christiani DC, Field JK, et al. (2012) Influence of common genetic variation on lung cancer risk: meta-analysis of 14 900 cases and 29485 controls. Hum Mol Genet 21: 4980-4995. 